\title{
Temperature dependence of domain-wall coercive field in magnetic garnet films
}

\author{
G. Vertesy \\ Institute of Physics, Czechoslovak Academy of Sciences, Na Slovance 2, CS-180 40 Praha 8, Czechoslovakia \\ and Central Research Institute for Physics, H-1525 Budapest, P. O. Box 49, Hungary \\ I. Tomás, L. Půst, and J. Pałes \\ Institute of Physics, Czechoslovak Academy of Sciences, Na Slovance 2, CS-180 40 Praha 8, Czechoslovakia
}

(Received 21 November 1991; accepted for publication 2 January 1992)

The coercive properties of magnetically uniaxial liquid-phase epitaxy garnet films were investigated between $10 \mathrm{~K}$ and the Neel temperature $\left(T_{N} \leqslant 500 \mathrm{~K}\right)$. Two independent methods, the results of which are nearly identical (magnetical response of oscillating domain walls and the method of coercive loops measurcd in a vibrating sample magnetometer), were used. Besides the usual domain-wall coercive field, $H_{\mathrm{dw}}$, the critical coercive pressure, $p_{\mathrm{dw}}$, was also introduced as it describes in a direct way the interactions of the domain walls with the wall-pinning traps. Both $H_{\mathrm{dw}}$ and $p_{\mathrm{dw}}$ were found to increase exponentially with decreasing temperature. Three different types of wall-pinning traps were identified in the sample and their strength, their rate of change with temperature, and their temperature range of activity were determined.

\section{INTRODUCTION}

Epitaxial magnetic garnet films belong to the most perfect single-crystalline materials, as their growth by liquidphase epitaxy (LPE) technology and the control of their magnetic properties are very well-established processes. Their magnetic domain structure is usually a very simple one: stripes or bubbles. Due to the large growth-induced uniaxial anisotropy, $K_{u}$, which keeps both the magnetization in the domains and the $180^{\circ}$ domain walls (DW) normal to the sample surface, there are no closure domains in the sample. This simple DW configuration makes it easy to investigate interactions of the moving DWs with the material, i.e., to investigate the domain-wall coercive field, $H_{\mathrm{dw}}$, the nonzero value of which originates from local variations of the DW energy as the walls sweep through the defects in the sample.

The domain-wall coercive field $H_{\mathrm{dw}}$ is defined as the minimum magnetic field normal to the sample surface which initiates an irreversible DW motion. A large number of theories (for a review see, e.g., Ref. 1) have attempted to calculate the coercive field from the material parameters and from an assumed distribution of the material defects. Some of the models met the experimental observations to a fair extent. The temperature dependence of $H_{\mathrm{dw}}$, however, has been studied only to a very limited extent. A considerable increase of the coercive field with decreasing temperature has usually been reported. ${ }^{2-8}$ The aim of the present paper is to measure the domain-wall coercive field, $H_{\mathrm{dw}}$, from the Néel temperature, $T_{N}$, down to about $T$ $=10 \mathrm{~K}$ and to suggest a mathematical expression describing the temperature dependence of $H_{\mathrm{dw}}$ within the whole temperature range of the existence of the ferrimagnetic order in our samples.

\section{EXPERIMENTAL RESULTS}

Measurements of two magnetic garnet films, $\mathrm{A}$ and $\mathrm{B}$, of chemical composition $\mathrm{Y}_{1.9} \mathrm{Sm}_{0.1} \mathrm{Ca}_{0.98} \mathrm{Fe}_{4.02} \mathrm{Ge}_{0.98} \mathrm{O}_{12}$ and $\mathrm{Y}_{1.53} \mathrm{Sm}_{0.27} \mathrm{Ca}_{0.96} \mathrm{Lu}_{0.24} \mathrm{Fe}_{4.01} \mathrm{Ge}_{0.99} \mathrm{O}_{12}$, respectively, grown by LPE on (111) GGG substrate are presented in this paper. The samples exhibited large uniaxial anisotropy which was perpendicular to the film plane, and superimposed over a small cubic one. The magnetization vector inside all domains was aligned perpendicular to the film plane. The basic parameters of the samples (see Table I) were determined at room temperature by standard methods for bubble garnet film characterization. ${ }^{9}$

Two methods for determining the coercive field, $H_{\mathrm{dw}}$, were used: (i) the low-frequency DW oscillation method ${ }^{10}$ ( $T_{i}$ between $90 \mathrm{~K}$ and $T_{N}$ ), and (ii) the method based on analyzing special minor hysteresis loops [domain-wall coercive loops (DWCL)] defined and described in Refs. 11 and 12 ( $T$ between 10 and $300 \mathrm{~K}$ ). The values of $H_{\mathrm{dw}}$ obtained by these two methods were equal to each other within the experimental error in the whole overlap of the two temperature ranges. (See Ref. 12 for a detailed analysis.)

(i) The low-frequency $(200-\mathrm{Hz})$ DW oscillation method $^{12}$ is based on the magneto-optical response of the domain system to an ac magnetic field perpendicular to the film plane. The ac field amplitude is slowly increased from zero and any DW motion is detected photoelectrically. The field amplitude corresponding to the extrapolated start of motion of the DW is interpreted as $H_{\mathrm{dw}}$.

These experiments were performed in special continuous-flow cryostat using the vapor of liquid nitrogen as a cooling fluid to cool the sample to about $90 \mathrm{~K}$. The sample could also be heated in the same holder to $500 \mathrm{~K}$. The temperature was measured by a Cu-constantan thermocouple. The use of this type of cryostat made it possible to observe the domain structure by a polarizing microscope in $\mathrm{dc}$ and/or ac applied magnetic fields. We confirmed by direct observation that mostly the maze-like stripe domain structure was kept in our samples during the measure- 
TABLE I. Properties of measured epitaxial garnet films at room temperature and their Néel temperature, $T_{N}$. Film thickness $h$; zero-field stripe period $P$, saturation magnetization $\mu_{0} M_{s}$ uniaxial anisotropy constant $K_{u}$; quality factor $Q$; characteristic length $l$.

\begin{tabular}{cccccccc}
\hline \hline Sample & $h$ & $P$ & $\mu_{0} M_{s}$ & $\begin{array}{c}K_{u} \\
\left(\mathrm{~J} / \mathrm{m}^{3}\right)\end{array}$ & $\begin{array}{c}Q \\
(1)\end{array}$ & $\begin{array}{c}l \\
(\mu \mathrm{m})\end{array}$ \\
\hline A & $(\mu \mathrm{m})$ & $(\mu \mathrm{m})$ & $\begin{array}{c}T_{N} \\
(\mathrm{~K})\end{array}$ & 4.2 & 0.47 & 463 \\
B & 5.3 & 9.0 & 19.7 & 640 & 4510 & 6.7 & 0.53 \\
\hline \hline
\end{tabular}

ments. At low temperatures irregular range domains were observed. ${ }^{13}$

(ii) The other method was based on magnetization measurements performed between 10 and $300 \mathrm{~K}$ on a vibrating sample magnetometer PAR Model 155 with a special helium cryostat. The magnetic moment of the sample was measured with the external magnetic field parallel to the easy magnetization axis, i.e., perpendicular to the film plane. The large signal coming from the paramagnetic GGG substrate as compared with that from the thin epitaxial film made it impossible to carry out reliable measurements below $10 \mathrm{~K}$.

Before each measurement of DWCL, the sample was carefully demagnetized by an ac magnetic field with the amplitude slowly decreasing to zero in order to ensure the starting configuration of each DWCL measurement to be the stripe domain structure with the lowest-energy equilibrium stripe period. The half-widths of the rhomboidshaped DWCL were interpreted as $H_{\mathrm{dw}}{ }^{12}$ As the internal field limits corresponding to the measurement of DWCLs were very close to zero (compared with the field necessary to saturate the sample), the domain walls were slightly moving perpendicular to their planes during the DWCL measurement, but no other changes in the domain structure (e.g., domain annihilation or a change of the domain period) took place. ${ }^{12}$ This was confirmed by direct observation of the domain structure. The $H_{\mathrm{dw}}(T)$ values measured by the two methods are presented in Fig. 1.

The pinning of domain walls on material imperfections described by the DW coercive field, $\mathbf{H}_{\mathrm{dw}}(T)$, can be also be characterized by the coercive pressure, $p_{\mathrm{dw}}(T)$, i.e., by the

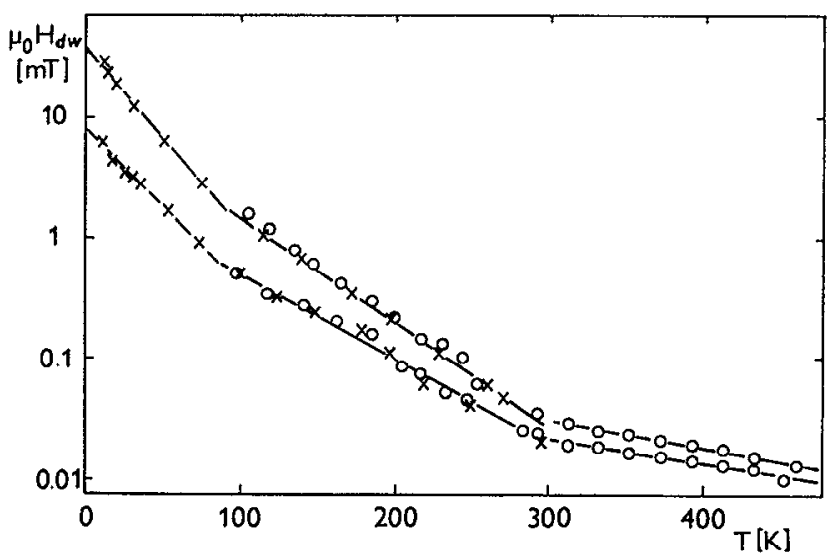

FIG. 1. Temperature dependence of domain wall coercive field, $H_{\mathrm{dw}}$, measured by $(X)$ cocrcive loops method, $(O)$ domain-wall oscillation method. critical force per unit area of DW which initiates an irreversible DW motion:

$$
p_{\mathrm{dw}}(T)=\mathbf{H}_{\mathrm{dw}}(T)\left[\mu_{0} \mathbf{M}_{1}(T)-\mu_{0} \mathbf{M}_{2}(T)\right],
$$

where $\mathbf{M}_{1}$ and $\mathbf{M}_{2}$ are magnetizations on both sides of the domain wall, $\left|\mathbf{M}_{1}\right|=\left|\mathbf{M}_{2}\right|=M_{s}(T)$. As in our case all the vectors $\mathbf{H}_{\mathrm{dw}}, \mathbf{M}_{1}$, and $\mathbf{M}_{2}$ are parallel and/or antiparallel to each other. The critical coercive pressure $p_{\mathrm{dw}}$ can be expressed simply as

$$
p_{\mathrm{dw}}(T)=2 H_{\mathrm{dw}}(T) \mu_{0} M_{s}(T) .
$$

The values of $p_{\mathrm{dw}}(T)$ according to (2) were plotted as a function of temperature in Fig. 2 in the semilogarithmic scale. The values $H_{\mathrm{dw}}(T)$ and $\mu_{0} M_{s}(T)$ used in (2) are those of Figs. 2 and 3. $\mu_{0} M_{s}(T)$ in Fig. 3 was independently measured by the vibrating sample magnetometer. The whole temperature range of the measurements could be divided into three linear parts of $p_{\mathrm{dw}}(T)$, viz., approximately between 10 and $90 \mathrm{~K}$, between 90 and $290 \mathrm{~K}$, and between 290 and $440 \mathrm{~K}$. A similar temperature dependence was found for both samples. The temperature dependence of $p_{\mathrm{dw}}$ can be described by the exponential function

$$
p_{\mathrm{dw}}=p_{\mathrm{dw} 0}^{i} \exp \left(-T / T_{i}\right)
$$

and/or

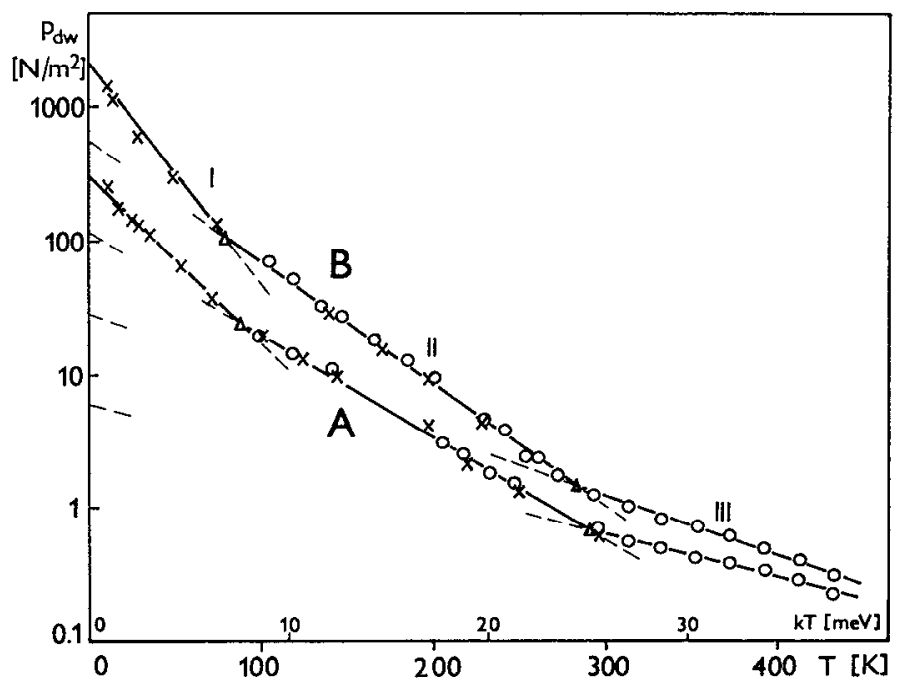

FIG. 2. Temperature dependence of critical pressure, $p_{\mathrm{d} w}$, measured by (i) domain-wall oscillation method $(O)$, and (ii) coercive loops method $(X)$ on samples $A$ and $B$. The temperature ranges of activity of the wall-pinning traps type I, II, and III differ slightly from sample to sample. The triangles show the limits of each range. Values of the extrapolated zero-temperature coercive pressures $p_{\mathrm{dwO}}^{i}$ are listed at the vertical axes. 


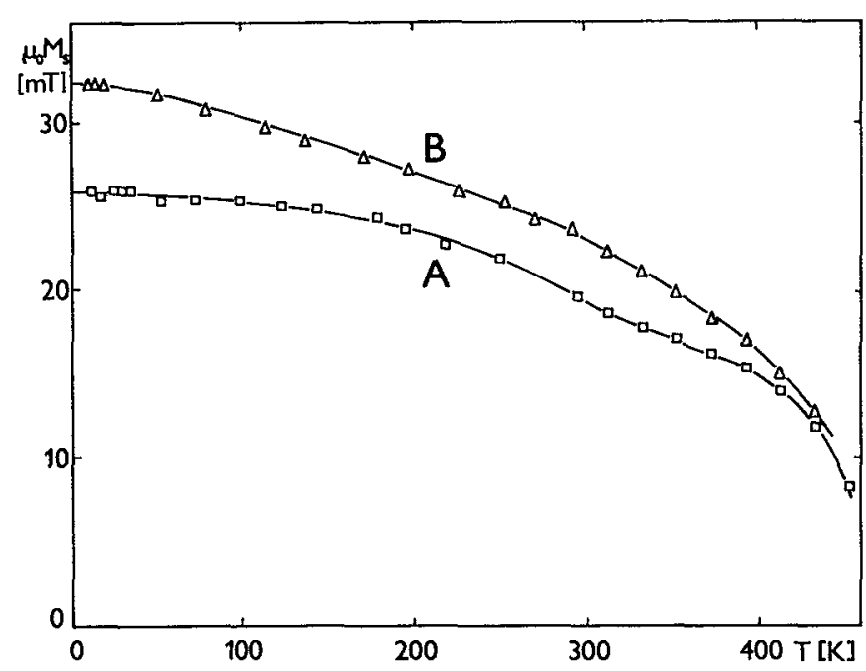

FIG. 3. Temperature dependence of saturation magnetization measured by vibrating magnetometer on samples $\mathrm{A}$ and $\mathrm{B}$.

$$
p_{\mathrm{dw}}=p_{\mathrm{dw} 0}^{i} \exp \left[-\left(k T / U_{i}\right)\right]
$$

in each of its linear parts, where $k$ is the Boltzmann constant and $p_{\mathrm{d} w 0}^{i}, T_{i}$, and $U_{i}$ are the characteristic pressure, characteristic temperature, and the characteristic energy, respectively, relevant to the $i$ th temperature range.

The values of $p_{\mathrm{dw} 0}^{i}, T_{i}$, and $U_{i}$ obtained by fitting (3) to the $p_{\mathrm{dw}}(T)$ data in the three temperature ranges for each sample are summarized in Table II. $T_{i}$ and $U_{i}$ values were found to be larger whereas $p_{\mathrm{dwo}}^{i}$ was smaller at higher temperature ranges than at the lower ones.

\section{DISCUSSION}

In order to interpret the temperature dependence of the coercive properties, we assume that the character of the domain structure and of the domain walls is not considerably modified even at low temperatures. Our preliminary experiments justify this assumption and show that just the stripe period increases and the width of the Bloch walls becomes narrower when the temperature decreases. The coercive behavior of thin epitaxial garnet films at low tcmperature is not very well known. An exponential decrease of the coercive field with increasing temperature has been reported on various materials by several authors. Carnegie and Claus ${ }^{2}$ fitted their coercive field values $H_{c}$ on PdFeMn to $H_{c} \sim \exp \left(U_{0} / k T\right)$ between 2 and $7 \mathrm{~K}$, where $U_{0}$ was a characteristics energy. Such $H_{c}$ dependence diverges at $T$
$=0 \mathrm{~K}$ and our data do not support such a curve. An exponential decrease of $H_{c}$ following a temperature dependence equivalent to (3) was reported on both soft ${ }^{3}$ and hard $^{8}$ magnetic materials. A dependence of the coercive field which is steeper at low temperatures than in a highertemperature range (similar to our results) has been observed by Komatsu et al. ${ }^{6}$ in amorphous Fe-Sm alloys.

Data measured on garnet films similar to those used in the present paper were analyzed in Ref. 7 on the basis of the theory of domain-wall pinning by a random array of uniform defects. ${ }^{5}$ A good fit was observed in the temperature range between 150 and $350 \mathrm{~K}$ in the form

$$
H_{\mathrm{dw}}^{1 / 2}=H_{x}^{1 / 2}-C T^{2 / 3} \text {, }
$$

where $H_{x}$ and $C$ were constants. At low temperatures, i.e., close to about $100 \mathrm{~K}$, the decrease of the coercivity was found to be stronger than the predicted $-T^{2 / 3}$ dependence; at higher temperatures a linear relation was found between the coercive field and temperature.

In most papers the domain-wall coercive field, $H_{\mathrm{dw}}$, is used as the characteristic parameter, but we decided to investigate the critical coercive pressure, $p_{\mathrm{dw}}$, on the basis of (1) and/or (2). Since the magnetization is actually the mediator of the push exerted by the applied field on the domain walls, the parameter $H_{\mathrm{dw}}$ includes the effect of the relative angle between the direction of the applied field, $\mathbf{H}_{\mathrm{dw}}$, and the magnetization vectors in the domains neighboring the wall, and it also includes the effect of the magnitude of the magnetization, $M_{s}(T)$.

In contrast to this, $p_{\mathrm{dw}}$ is actually "normalized" with respect to both the direction and the magnitude of the magnetization. In our opinion, the critical coercive pressure, $p_{\text {dw }}$, describes better the microphysical mechanism of the domain-wall pinning at the material defects as it simply represents the force needed to act on a unit area of DW in order to tear it off the pinning trap.

The advantage of the $p_{\mathrm{dw}}$ description over the $H_{\mathrm{dw}}$ description is most evident in the temperature regions where $M_{s}(T)$ changes rapidly. This is the case, for instance, with numerous types of magnetic garnets having a compensation point close to that point. In the vicinity of the compensation point, $M_{s}(T)$ approaches zero, $H_{\mathrm{dw}}$ diverges to infinity, but $p_{\mathrm{dw}}(T)$ passes continuously through this singularity (see Ref. 13).

Figure 2 illustrates the principal experimental results of the present paper. In general, it shows that whatever the origin of the wall-pinning defects in the samples, their "strength" decreases with increasing temperature in accor-

\begin{tabular}{|c|c|c|c|c|c|c|c|c|c|}
\hline \multirow[b]{2}{*}{ Sample } & \multicolumn{3}{|c|}{$\begin{array}{c}\mathrm{I} \\
10-90 \mathrm{~K}\end{array}$} & \multicolumn{3}{|c|}{$\underset{90-290 \mathrm{~K}}{\text { II }}$} & \multicolumn{3}{|c|}{$\underset{290-440 \mathrm{~K}}{\text { III }}$} \\
\hline & $\underset{\left(\mathrm{N} / \mathrm{m}^{2}\right)}{p_{\mathrm{dw0}}^{\mathrm{I}}}$ & $\begin{array}{c}U_{\mathrm{I}} \\
(\mathrm{meV})\end{array}$ & $\begin{array}{c}T_{\mathrm{I}} \\
(\mathrm{K})\end{array}$ & $\underset{\left(\mathrm{N} / \mathrm{m}^{2}\right)}{p_{\mathrm{d} w 0}^{\mathrm{II}}}$ & $\underset{(\mathrm{meV})}{U_{\mathrm{II}}}$ & $\begin{array}{c}T_{\mathrm{II}} \\
(\mathrm{K})\end{array}$ & $\underset{\left(\mathrm{N} / \mathrm{m}^{2}\right)}{p_{\mathrm{d} 0}^{\mathrm{III}}}$ & $\underset{(\mathrm{meV})}{U_{\mathrm{III}}}$ & $\begin{array}{l}T_{\mathrm{III}} \\
(\mathrm{K})\end{array}$ \\
\hline A & 300 & 3.03 & 35.2 & 118 & 4.92 & 57.1 & 6.0 & 11.15 & 129.4 \\
\hline B & 2050 & 2.31 & 26.8 & 570 & 4.11 & 47.7 & 26.5 & 8.42 & 97.7 \\
\hline
\end{tabular}

TABLE II. Parameters $p_{\mathrm{dw} 0+}^{i} \mathrm{~T}_{\mathrm{i}}$, and $U_{i}$ obtained from fitting of coercive pressure $p_{\mathrm{dw}}(T)$ (see Fig. 2) to expression (3). 
dance with the plotted curve $p_{\mathrm{dw}}(T)$. However, the existence of the two distinct breaking points of the semilogarithmic plot requires some explanation. We shall introduce three possible explanations for the existence of the breaking points.

First, it is necessary to decide whether the breaking points are really connected with the measured material or with some kind of instrumental effect due to the measurement methods employed. The necessity to answer this question is strongly supported by the fact that the lower breaking point is close to the temperature of liquid nitrogen (i.e., close to the limit of applicability of the optical method) and the higher one is not far from room temperature (i.e., close to the point where cooling of the sample was changed for its heating). Even though these indications may give rise to some suspicion, we do not consider the breaking points to originate from the experimental procedure. One of the reasons for this is the fact that both breaking points appeared even on those $p_{\mathrm{dw}}(T)$ curves which were measured continuously through the critical temperature without any experimental change. In particular, if the lower breaking point is considered, both samples were measured through this point in the vibrating sample magnetometer while being cooled by evaporated helium gas from about $10 \mathrm{~K}$ to room temperature (crosses in Figs. 1 and 2). In conclusion, we consider the breaking points to be really connected with the sample, i.e., with the behavior of the wall-pinning defects in the material.

The other reason which shows that the breaking points do not originate from the experimental procedure is that for other samples not shown in this paper (which were not measured in the full temperature range of the ferrimagnetic phase but only in the 90-300 $\mathrm{K}$ range), the higher breaking points were found at different temperatures $(248,250,255$, and $265 \mathrm{~K}$ ). This means that the breaking points can be considered to be some kind of material parameter, which characterizes the temperature dependence of the coercive properties. This parameter is different for different samples.

This second possible explanation is simply that it is an inherent property of the pinning defects that they behave as observed, i.e., that their "strength" really decreases with temperature following three different experimental functions, one after the other. This may be either the property of each of the pinning defects as of separate entities, or the breaks at $p_{\mathrm{dw}}(T)$ could appear as the marking points of a mutual interaction when-due to the increased temperature-the potential wells of the defects become greater and their slopes start to overlap each other. There are, however, three qualitative objections to the mutual interaction idea: (1) It is possible to explain one breaking point by the start of mutual overlapping of the potential wells, but two can hardly be expected in a set of mutually equivalent defects. (2) The overlapping of potential wells should eventually lead to less localized pinning traps, i.e., the walls should be allowed to move more and more reversibly. This is in contrast with what is actually observed during the DWCL measurement up to the Néel temperature. (The DWCLs as defined in Ref. 12 and modeled in
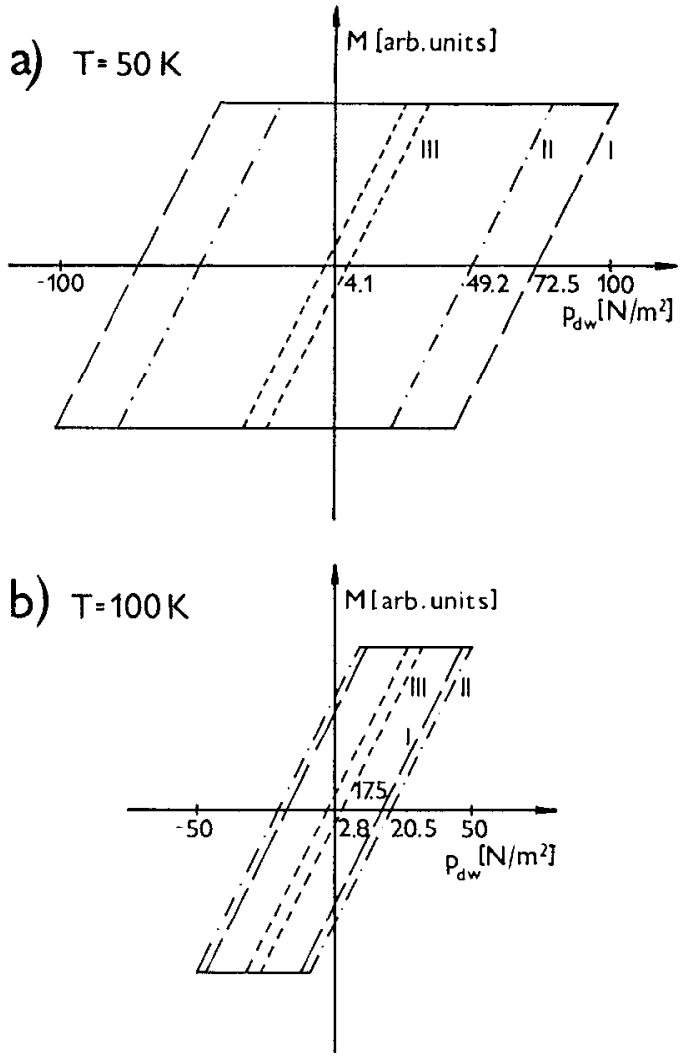

FIG. 4. Coercive loops of the three types of wall pinning traps in sample A. (a) $T=50 \mathrm{~K}: p_{\mathrm{dw}}^{(1)}=72.5 \mathrm{~N} / \mathrm{m}^{2}, p_{\mathrm{dw}}^{(2)}=49.2 \mathrm{~N} / \mathrm{m}^{2}, p_{\mathrm{dw}}^{(3)}=4.1 \mathrm{~N} / \mathrm{m}^{2}$. The wall-pinning traps I are active, traps II and III are inactive. The measurement results with coercive loop I. (b) $T=100 \mathrm{~K}: p_{\mathrm{dw}}^{(1)}=17.5$ $\mathrm{N} / \mathrm{m}^{2}, p_{\mathrm{dw}}^{(2)}=20.5 \mathrm{~N} / \mathrm{m}^{2}, p_{\mathrm{dw}}^{(3)}=2.8 \mathrm{~N} / \mathrm{m}^{2}$. Wall-pinning traps II are active, traps I and II are inactive. The measurement results with the coercive loop II.

Ref. 14 are proof of the existence of strict localization of the pinning traps.) (3) The overlapping of the potential wells should cause a more rapid decrease of their slopes than before they started to overlap. Just the contrary is observed: the decrease of $p_{\text {dw }}$ (i.e., the decrease of the critical slope of the potential wells) is less rapid at higher temperatures. Even though we cannot completely exclude the explanation of the breaking points through an inherent property of a set of mutually equivalent defects, we are not inclined to accept it.

We tend to accept the third possible explanation which is based on the presumed presence of three sets of mutually nonequivalent wall-pinning defects in the investigated LPE garnet films. Our assumption is that the defects are of mutually different quality, different strength, and different temperature dependence of their strength. Despite knowing nothing about their real origin, we can try to explain their properties in the following way.

Any set of a large number of mutually equivalent, localized wall-pinning defects in a otherwise uniform magnetic material (or at least LPE garnet films) makes it presence evident in a careful magnetic measurement through a characteristic coercive loop. ${ }^{11,12}$ The loop is rhomboid shaped and its half-width is $H_{\mathrm{dw}}$ when measured and/or $p_{\mathrm{dw}}$ when recalculated according to $(2)$. If there are three 
different sets of defects in the sample, and the critical coercive pressure $p_{\mathrm{dw}}$ of each set is known, the coercive loop of each set of defects can be drawn. Figure 4(a) shows such a schematical drawing for sample $A$ at $T=50 \mathrm{~K}$. But whereas the coercive loops of each set of defects can be drawn, only that with the largest $p_{\mathrm{dw}}$ can be measured: The measurement of sample A at $T=50 \mathrm{~K}$ results in loop I only because the set of defects $I$ is characterized by the largest critical coercive pressure of all [Eq. (3a): $\left.p_{\mathrm{dw}}^{\mathrm{I}}(T=50 \mathrm{~K})=p_{\mathrm{dw} 0}^{\mathrm{I}} \exp \left(-50 / T_{I}\right)=72.5 \mathrm{~N} / \mathrm{m}^{3}\right]$, and the walls can in fact be pinned solely by the pinning traps with the greatest "strength."

If the temperature is increased, the strength (i.e., the critical coercive pressure, $p_{\mathrm{dw}}$ ) of each type of pinning traps decreases in accordance with (3) but the parameters in Eq. (3) are different for each type. As can be seen from the parameters listed in Table II, the "fatter" the DWCL at $T=50 \mathrm{~K}$ in Fig. 4, the faster it gets "slimmer" with increasing temperature. In this way we can understand the breaking points in the $p_{\mathrm{dw}}(T)$ plot to correspond to the "taking over" of the DWCL of one type of defect by the next type. Figure 4(b) illustrates the situation at $T=100$ $\mathrm{K}$ shortly after (at $T \approx 86 \mathrm{~K}$ ) the pinning traps of type II took over from the I. A similar takeover by type III from type II takes place at $T \approx 292 \mathrm{~K}$.

We consider the above explanation of the two observed breaking points in the $p_{\mathrm{dw}}(T)$ plot via the presumed existence of three different types of wall pinning traps in the investigated magnetic materials, to be quite a challenge. It opens the way for theoretical models of material defects which can give rise to wall-pinning traps with the observed properties and at the same time it asks for further experiments on the domain-wall pinning: influencing the pinning traps by means other than by slow temperature changes (strain, annealing), introduction of pinning traps of a new type (high-energy ion implantation, laser heating), and/or investigation of the distribution density of the pinning traps close to and far from the breaking points on the $p_{\mathrm{dw}}(T)$ curve.

The rate of change of $p_{\mathrm{dw}}(T)$ in (3) can be expressed either by a characteristic temperature $T_{i}(3 \mathrm{a})$ or by a characteristic energy $U_{i}$ (3b) (see Table II for the list of $T_{i}$ and $U_{i}$ values). Even though it is possible to ascribe a value of $U_{i}$ to each temperature interval, it is difficult to find its physical meaning. The values of $U_{i}$ of a few $\mathrm{meV}$ $\left(\cong 10^{-22} \mathrm{~J}\right)$ can hardly mean fluctuations of the domainwall energy density (typically the domain-wall energy $\sigma_{w} \cong 10^{-4} \mathrm{~J} / \mathrm{m}^{2}$ ) as the wall drops in the pinning traps.

\section{CONCLUSIONS}

Coercive properties of two samples of typical LPE single-crystal films of magnetic garnets were investigated in the temperature range from $10 \mathrm{~K}$ up to the Néel temperature $(\approx 500 \mathrm{~K})$. Critical coercive pressure, $p_{\mathrm{dw}}$ was defined as the net force per unit area of the domain wall $\left(\mathrm{N} / \mathrm{m}^{2}\right)$ needed to initiate an irreversible motion of the wall, i.e., to tear the wall off a wall-pinning trap in the material. The critical coercive pressure, $p_{\mathrm{dw}}$, was found to be a more straightforward means of describing any domain-wall material defect interaction than the domain-wall coercive field, $H_{\mathrm{dw}}$, as the former is normalized with respect to the magnitude and direction of magnetization.

The measured $p_{\mathrm{dw}}(T)$ plot shows an exponential decrease with increasing temperature. Three different temperature regions with different slopes of the exponential drop of $p_{\mathrm{dw}}(T)$ were observed for each sample. In explanation, three different sets of wall-pinning traps were suggested to coexist in the sample, each of them being active in one of three different temperature regions. The breaking points on the semilogarithmic plot of $p_{\mathrm{dw}}(T)$ were identified with the limits of activity of one type of wall-pinning traps and taking over of the next type. The material constant characterizing the strength [i.e., the critical coercive pressure extrapolated down to $\left.T=0 \mathrm{~K}, p_{\mathrm{dwo}}^{(i)}\right]$ and the rate of change of the strength with temperature (characteristic temperature $T_{i}$ and/or energy $U_{i} / k T_{i}$ ) were determined for each type of wall-pinning traps in both samples and listed in Table II.

Theoretical models of the wall-pinning traps with the properties as found by the described measurement are needed. New experiments which would investigate the external influence on the wall-pinning traps (i.e., the material coercivity) and would better describe their distribution are tentatively suggested. Similar investigations would be valuable on materials where the coercivity and wall pinning play an important role in their technical use (hysteresis losses).

\section{ACKNOWLEDGMENT}

The authors are grateful to L. Murtinová for her valuable remarks concerning the lucidity of the explanations.

'M. Pardavi-Horváth, IEEE Trans. Magn. MAG-21, 1694 (1985)

${ }^{2}$ D. W. Carnegie, Jr. and H. Claus, Phys. Rev. B 20, 1280 (1979).

${ }^{3}$ D. A. Read, T. Moyo, and G. C. Hallam, J. Magn. Magn. Mater. 44, 279 (1984); 54-57, 309 (1986).

${ }^{4}$ D. G. Rancourt, S. Chehab, and G. Lamarche, J. Magn. Magn. Mater. 78, 129 (1989).

${ }^{5}$ P. Gaunt, Philos Mag. B 48, 261 (1983).

${ }^{6}$ J. Maguin, H. Jouve, and B. Barbara, J. Appl. Phys. 50, 1538 (1979).

${ }^{7}$ M. Pardavi-Horváth and G. Vértesy, J. Appl. Phys. 58, 3827 (1985).

${ }^{8}$ H. Komatsu, K. Fukamichi, N. Saito, and Y. Nakagawa, IEEE Trans. J. Magn. Jpn. 4, 270 (1989).

${ }^{9}$ R. M. Josephs, AIP Conf. Proc. 10, 286 (1972).

${ }^{10}$ J. A. Seitchik, G. K. Goldberg, and W. D. Doyle, J. Appl. Phys. 42, 1272 (1971).

${ }^{11}$ L. Pủst, I. Tomás̆, and G. Vértesy, Proceedings of the European Magnetic Materials Applied Conference, Rimini, 1989, p. 176.

${ }^{12} \mathrm{G}$. Vértesy, L. Pủst, and I. Tomáš, J. Phys. D 24, 1482 (1991).

${ }^{13}$ B. Antonini, C. Borghese, S. Menegalli, P. Paroli, R. Tappa, and M. Toni, J. Appl. Phys. 48, 3864 (1977).

${ }^{14}$ I. Tomáš, J. Magn. Magn. Mater. 87, 5 (1990). 